\title{
Estudo da Repetitividade de Classes de Compostos em Espectros de Massas por ESI(+) FT-ICR MS para um Óleo Pesado
}

\author{
Victor Gustavo K. Cardoso, Thales R. Rosa, Wanderson Romão \& \\ Paulo R. Filgueiras
}

A espectrometria de massas surgiu como uma excelente alternativa para caracterizar o petróleo e suas frações. No entanto, é necessário verificar os resultados obtidos através de parâmetros como a repetitividade. Foram obtidos espectros de massas de um óleo pesado no modo positivo em triplicata. Foi calculado o erro padrão de cada classe de compostos e o ruído foi variado entre 1 e $10 \%$, para relacioná-lo com o erro padrão. Foram encontradas as classes de compostos $\mathrm{N}, \mathrm{NS}, \mathrm{NS}_{2}$ e $\mathrm{O}_{3}$ no óleo. Os resultados obtidos foram satisfatórios. A classe $\mathrm{O}_{3}$ apresentou a melhor concordância dos resultados e a classe $\mathrm{NS}_{2}$ apresentou a melhor correlação entre o ruído e o erro padrão.

Palavras Chave: Espectrometria de Massas; Repetitividade; Petróleo; Metrologia; Petroleômica; Química Analítica.

Mass spectrometry emerged as an excellent alternative to characterize oil and its fractions. However, it is necessary to verify the results obtained through parameters such as repeatability. Mass spectra of a heavy oil were obtained in the positive mode in triplicate. The standard error of each class of compounds was calculated and the noise was varied between 1 and $10 \%$ to relate it to the standard error. The classes of compounds $\mathrm{N}, \mathrm{NS}, \mathrm{NS}_{2}$ and $\mathrm{O}_{3}$ in the oil were found. The results were satisfactory. The $\mathrm{O}_{3}$ class presented the best agreement of the results and the class $\mathrm{NS}_{2}$ presented the best correlation between the noise and the standard error.

Keywords: Mass Spectrometry; Repeatability; Petroleum; Metrology; Petroleomics; Analytical Chemistry. 


\section{Introdução}

O petróleo é uma mistura extremamente complexa de compostos orgânicos. Sua constituição pode variar de acordo com a matriz geradora e sua evolução térmica, garantindo uma variedade infinita de óleos. ${ }^{1}$

Sua caracterização físico-química é fundamental para todo o processo industrial, desde a extração, avaliação e refino, além da importância econômica e ambiental. A caracterização de óleos brutos e seus derivados é algo crescente na indústria, pois a quantificação de compostos contendo nitrogênio, oxigênio e enxofre é de grande interesse a fim de aperfeiçoar processos, reduzir custos e contornar problemas de corrosão. ${ }^{2,3}$

Os métodos tradicionais para caracterização físicoquímica um óleo bruto e seus derivados uma especiação das classes de compostos presentes no petróleo, além do bom resultado, rapidez e baixo custo das análises. ${ }^{4,5}$

A introdução de espectrometria de massas de altíssima resolução e exatidão (FT-ICR MS) tem se mostrado muito eficiente no processo de caracterização de óleos brutos, permitindo a separação e identificação dezenas de milhares de componentes no óleo bruto com muita eficiência e rapidez. ${ }^{6,7}$

Entretanto, por se tratar de uma técnica de alta sensibilidade, é necessário avaliar a qualidade espectral, pois quaisquer erros e incertezas afetam o resultado e é impossível realizar uma análise química livre de erros e incertezas, pois eles são intrínsecos às medições. ${ }^{8}$ Uma das formas de lidar com os erros é com a avaliação da repetitividade de um método de análise, que é o grau de concordância entre os resultados de medições sucessivas de um mesmo mensurando, efetuadas sob as mesmas condições de medição. ${ }^{9}$

$\mathrm{Na}$ tentativa de mensurar os erros, este estudo buscou avaliar o erro padrão do número de componentes das classes identificadas em função da intensidade mínima e buscar a relação entre o erro padrão e a abundância mínima dos sinais de espectros de massas de altíssima resolução e exatidão de um petróleo pesado.

\section{Metodologia}

Para a realização do experimento foi utilizada uma amostra de petróleo pesado. O preparo das amostras foi dado da seguinte forma: A amostra de petróleo foi homogeneizada e pesada uma alíquota de $1 \mathrm{mg}$ do óleo, que foi dissolvido em $2 \mathrm{~mL}$ de uma mistura de tolueno e metanol 1:1, sendo homogeneizada em seguida. Foi, então, adicionado $1 \mu \mathrm{L}$ de ácido fórmico PA para protonação das espécies presentes na amostra, para obter o espectro no modo positivo. O procedimento descrito foi realizado em triplicata real, obtendo um total de três espectros para a amostra. Depois da preparação das amostras, foi utilizado o espectrômetro de massas de altíssima resolução e exatidão (FT-ICR-MS) da Bruker ${ }^{\circledR}$ para a obtenção dos espectros de cada uma das amostras, que foram obtidos sob os mesmo parâmetros de obtenção. Posteriormente, foi criada uma lista externa de séries homólogas para auxiliar no processamento dos espectros, que foi feito utilizando o software Composer da Sierra Analytics ${ }^{\circledR}$, onde foram obtidas as classes de compostos presente no óleo. Os espectros foram processados mantendo sempre os mesmos parâmetros de calibração, variando apenas a abundância mínima dos sinais do espectro de $1 \%, 2 \%, 3 \%$, $4 \%, 5 \%$ e $10 \%$. A partir dos dados processados, foi então realizado o tratamento estatístico.

\section{Resultados e Discussão}

Depois do preparo das amostras de acordo com a metodologia descrita, foi obtido o espectro de massas do óleo estudado no modo positivo. O perfil espectral de uma dos ensaios realizados pode ser observado na Figura 1. Todos os espectros apresentaram perfis muito similares.

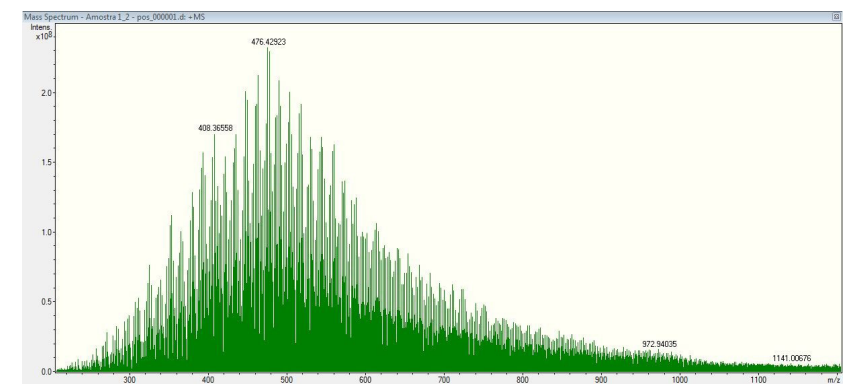

Figura 1. Espectro de massas de um petróleo pesado no modo positivo. 
Um espectro de massas de um petróleo é extremamente complexo para ser analisado diretamente. Para isso, é necessário utilizar softwares que possam interpretar os dados do espectro. Foi utilizado, então, o software Composer, da Sierra Analitics ${ }^{\circledR}$. O processamento de espectros de massas atribui fórmulas químicas para os sinais de massas obtidos no espectro, onde apresentam a probabilidade daquele sinal ser daquele composto e o seu respectivo erro. Esse processamento é muito eficiente, devido a sua altíssima resolução e exatidão, podendo diferenciar dois compostos de massas muito próximas apenas pela diferença de massas nas casas decimais. O processamento atribui o sinal ao determinado composto e agrupa os compostos por classes. A classe de compostos é o agrupamento de compostos orgânicos que apresentam heteroátomos em comum, por exemplo, os compostos de classe $\mathrm{N}$ são compostos orgânicos que apresentam um único átomo de nitrogênio como heteroátomo, os compostos de classe NS apresentam um átomo de nitrogênio e um átomo de enxofre como heteroátomos e a classe $\mathrm{O}_{3}$ apresentam três átomos de oxigênio como heteroátomos.

Através do processamento dos dados, foi possível encontrar quatro classes de compostos presente no petróleo: $\mathrm{N}, \mathrm{NS}, \mathrm{NS}_{2} \mathrm{e}_{3}$. O processamento foi realizado variando a abundância mínima dos sinais em 1\%, 2\%, 3\%, 4\%, 5\% e $10 \%$. A variação da abundância mínima baseou-se na ideia de verificar o comportamento do erro padrão de acordo com a variação da abundância mínima. Para cada classe de compostos foram calculadas as médias da quantidade de compostos encontrados e os erros padrão. As informações podem ser observadas na Figura 2.

Observa-se que a quantidade de compostos assimilados no processamento reduz de acordo com o aumento da abundância mínima, como o esperado, mas torna-se necessário reforçar isso para mostrar que não houve erro de assimilação dos sinais. Ao analisar as barras de erros, é perceptível que o erro padrão da classe $\mathrm{O}_{3}$ é muito menor quando comparado às classes $\mathrm{N}, \mathrm{NS}$ e $\mathrm{NS}_{2}$, principalmente na abundância mínima de $1 \%$. O maior erro padrão nesta classe se apresentou na abundância mínima de $10 \%$.
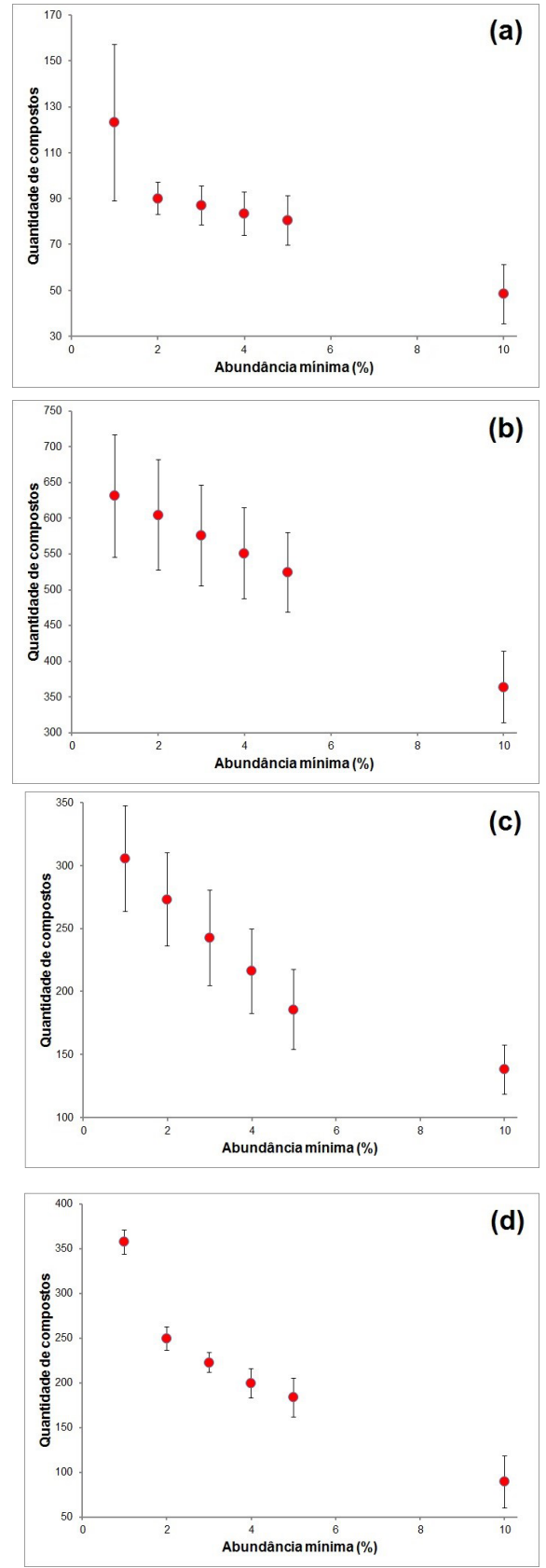

Figura 2. Quantidade média de compostos encontrados nas classes (a) $\mathrm{N}$, (b) NS, (c) $\mathrm{NS}_{2}$ e (d) $\mathrm{O}_{3}$ em função da abundância mínima dos sinais e seus respectivos erros padrão. 
Para observar alguma tendência na relação entre os erros padrão e a abundância mínima, os dois foram plotados e pode ser observado na Figura 3.

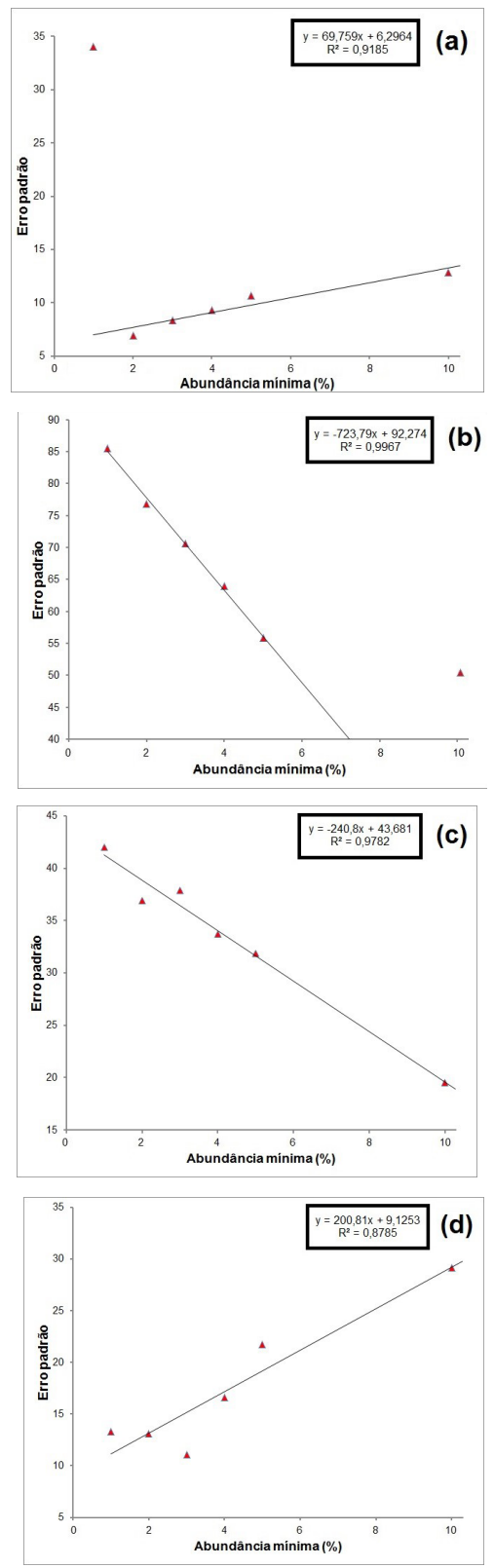

Figura 3. $\mathrm{O}$ erro padrão das classes (a) $\mathrm{N}$, (b) $\mathrm{NS}$, (c) $\mathrm{NS}_{2}$ e (d) $\mathrm{O}_{3}$ em função da abundância mínima dos sinais.
Verificamos a ocorrência de uma relação entre o erro padrão e a abundância mínima dos sinais. Esperava-se que o erro padrão reduzisse a medida que a intensidade mínima aumentasse, apresentando uma relação decrescente. Em geral, as relações se mostram variadas. Nos compostos da classe $\mathrm{NS}_{2}$, apresenta-se um coeficiente de determinação de aproximadamente 0,98 . Nos compostos de classe $\mathrm{N}$ e classe NS foi necessário desconsiderar o erro padrão para a abundância de $1 \%$ e $10 \%$, respectivamente, pois os valores se apresentavam muito discrepantes, aumentando o coeficiente de determinação. O coeficiente de determinação da classe $\mathrm{O}_{3}$ foi o menor, mas apresentando um valor razoável.

\section{Conclusões}

Por meio do estudo, foi possível avaliar a concordância entre os dados das triplicatas das análises de petróleo por espectrometria de massas de altíssima resolução e exatidão (FT-ICR MS). A classe $\mathrm{O}_{3}$ apresentou valores de erro padrão bastante satisfatórios para as abundâncias mínimas entre 1 e $5 \%$. A classe $\mathrm{N}$ apresentou um erro padrão relativamente alto em $1 \%$, mas com o aumento da intensidade mínima, o erro padrão reduz consideravelmente. A relação entre o erro padrão e a intensidade mínima não mostraram uma tendência decrescente para todas as classes, sendo que apenas a classe $\mathrm{NS}_{2}$ mostrou uma linearidade muito boa (com coeficiente de determinação de 0,98 ) considerando todos os erros padrão. No geral, todas as classes apresentaram uma relação razoável.

\section{Agradecimentos}

Primeiramente agradeço a Deus. Agradeço à UFES e ao LabPetro por terem permitido a realização dos trabalhos. Agradeço à FAPES pelo financiamento do projeto PIBITI.

\section{Referências Bibliográficas}

1. Thomas, J. E. Fundamentos da engenharia do petróleo. Rio de Janeiro: Editora Interciência, 2001. 271 p.

2. Rodgers, R. P., Schaub, T. M., Marshall, A. G. Petroleomics: MS 
Return to Its Roots. American Chemical Society. 2005. 1. 20-27 .

3. Filgueiras, P. R. Determinação da composição de blends de petróleos utilizando FTIR-ATR e calibração multivariada. 2011. $113 \mathrm{f}$.

Dissertação (Mestrado em Química). Departamento de Química, Universidade Federal do Espírito Santo, Vitória, 2011.

4. Dias, J. C. M. Estudo exploratório das propriedades de caracterização de petróleos brasileiros. Dissertação de Mestrado. Departamento de Química da Universidade Federal do Rio de Janeiro, Rio de Janeiro, 2005.

5. Rainha, K. P. Estudo de alguns parâmetros físico-químicos de petróleos utilizando modelagem multivariada associada a espectroscopia no infravermelho. Dissertação de Mestrado. Departamento de Química, Universidade Federal do Espírito Santo, Vitória, 2014

6. Marshall, A. G.; Rodgers, R. P. Petroleomics: The Next Grand Challenge for Chemical Analysis Petroleum and Mass Spectrometry: Divergent. Acconts of Chemical Research. 2004, 37. 53-59.

7. Marshall, A. G.; Rodgers, R. P. Petroleomics: Chemistry of the Underworld. PNAS. 2008, 105. 18090-18095.

8. Skoog, D. A., West, D. M.; Holler, F.J.; Crouch, S. R. Fundamentos de Química Analítica. $8^{\mathrm{a}}$. São Paulo: Cengage Learning, 2006. 1000 p.

9. Lira, F. A. Metrologia Na Indústria. $3^{\text {a }}$. São Paulo: Editora Érica, 2004. $246 \mathrm{p}$.

\section{Victor Gustavo K. Cardoso 1*, Thalles R. Rosa', Wanderson Romão ${ }^{1,2}$ \& Paulo R. Filgueiras $^{1}$}

\footnotetext{
1. Instituto de Química, Universidade Estadual de Campinas - Unicamp. Rua Josué de Castro, 126 - Cidade Universitária - 13083-861, Campinas - SP, Brasil.

2. Instituto Federal do Espírito Santo - IFES, Campus Aracruz. Avenida Morobá, 248 - Morobá - 29192-733, Aracruz - ES, Brasil.

3. Departamento de Química, Centro de Ciências Exatas, Universidade Federal do Espírito Santo - UFES. Avenida Fernando Ferrari, 514 Goiabeiras - 29075-910, Vitória - ES, Brasil.

4. Instituto Federal do Espírito Santo - IFES, Campus Vila Velha. Avenida Ministro Salgado Filho, 1000 - Soteco - 29106-010, Vila Velha - ES, Brasil.

*E-mail: victor.cardoso@aluno.ufes.br
} 